\title{
Dynamics of nonequilibrium electrons on neutral center states of interstitial magnesium donors in silicon
}

\author{
S. G. Pavlov, ${ }^{1}$ N. Deßmann, ${ }^{2}$ A. Pohl, ${ }^{2}$ V. B. Shuman, ${ }^{3}$ L. M. Portsel, ${ }^{3}$ A. N. Lodygin, ${ }^{3}$ Yu. A. Astrov, ${ }^{3}$ S. Winnerl, ${ }^{4}$ \\ H. Schneider, ${ }^{4}$ N. Stavrias, ${ }^{5}$ A. F. G. van der Meer, ${ }^{5}$ V. V. Tsyplenkov, ${ }^{6}$ K. A. Kovalevsky, ${ }^{6}$ R. Kh. Zhukavin, ${ }^{6}$ V. N. Shastin, ${ }^{6}$ \\ N. V. Abrosimov, ${ }^{7}$ and H.-W. Hübers ${ }^{1,2}$ \\ ${ }^{1}$ Institute of Optical Sensor Systems, German Aerospace Center (DLR), Berlin, Germany \\ ${ }^{2}$ Department of Physics, Humboldt Universität zu Berlin, Berlin, Germany \\ ${ }^{3}$ Ioffe Institute, Russian Academy of Sciences, St. Petersburg, Russia \\ ${ }^{4}$ Helmholtz-Zentrum Dresden-Rossendorf, Dresden, Germany \\ ${ }^{5}$ Radboud University, Institute of Molecules and Materials, FELIX Laboratory, Nijmegen, The Netherlands \\ ${ }^{6}$ Institute for Physics of Microstructures, Russian Academy of Sciences, Nizhny Novgorod, Russia \\ ${ }^{7}$ Leibniz Institute for Crystal Growth, Berlin, Germany \\ (Received 21 April 2016; revised manuscript received 8 June 2016; published 29 August 2016)
}

\begin{abstract}
Subnanosecond dynamics of optically excited electrons bound to excited states of neutral magnesium donor centers in silicon has been investigated. Lifetimes of nonequilibrium electrons have been derived from the decay of the differential transmission at photon energies matching the intracenter and the impurity-to-conduction band transitions. In contrast to hydrogenlike shallow donors in silicon, significantly longer lifetimes have been observed. This indicates weaker two-phonon and off-resonant interactions dominate the relaxation processes in contrast to the single-intervalley-phonon-assisted impurity-phonon interactions in the case of shallow donors in silicon.
\end{abstract}

DOI: 10.1103/PhysRevB.94.075208

\section{INTRODUCTION}

Magnesium (Mg, group-IIA) atoms in interstitial positions of a silicon $(\mathrm{Si})$ host lattice form double donor centers with a binding energy $E_{\mathrm{i}}$ in the neutral $\mathrm{Mg}^{0}$ state of $\sim 107 \mathrm{meV}$ [1]. The so-called "chemical shift" of a neutral $\mathrm{Mg}^{0}$ donor, i.e., the downshifting of the impurity ground state relative to its position as derived from effective mass theory [1], is larger than the group- $\mathrm{V}$ donors in $\mathrm{Si}$. This makes magnesium in $\mathrm{Si}$ a transition case between the shallow donors in $\mathrm{Si}$, where singleintervalley-phonon-assisted transitions dominate intracenter relaxation [2,3], and the deeper, heliumlike group-VI donors in $\mathrm{Si}$, where electron capture into the ground state can be assisted only by multiphonon interactions [4]. In contrast to direct band-gap semiconductors, where interactions with zonecentered optical phonons and their overtones are most intense, in multivalley crystals scattering between equivalent valleys often dominates over other electron-phonon interactions. At intracenter-phonon resonances such transitions happen almost as fast as those involving higher-energy optical modes [2]. But even far from such resonances these interactions cause typical intracenter lifetimes of hydrogenlike donors in $\mathrm{Si}$ not longer than 150-230 ps $[5,6]$. Note that the binding energies of even-parity excited states in Si: $\mathrm{Mg}^{0}$ [Fig. 1(a)] have not yet been derived directly from equilibrium spectroscopy. Because of this uncertainty one cannot exclude an intermediate case between the single-phonon relaxation scheme (assuming the binding energy of the lowest excited state to be $52.5 \pm 3 \mathrm{meV}$ [1]) and the two-phonon relaxation scheme, if the binding energy of the lowest excited state is $\approx 33 \mathrm{meV}$ (common to all other donors) [7]. Two-phonon interactions in silicon give rise to dominant bands in lattice absorption spectra [8] as well as in Raman spectra of Si [9]. The cut-off energy for two-phonon interactions in Si exceeds the binding energy of the neutral magnesium donor. Therefore, one would expect the occurrence of related electron scattering.
The question of whether the multiphonon interaction process can result in either fast or relatively slow intracenter relaxation in $\mathrm{Si}: \mathrm{Mg}^{0}$ is the main focus of this work. The knowledge of the intracenter dynamics of magnesium related centers is of great importance for potential photonic application of such a material in the mid- and far-infrared wavelength ranges [10].

Here we report on theoretical calculations and direct measurements of the dynamics of nonequilibrium electrons in the conduction band continuum as well as in odd-parity excited states of neutral magnesium donors in $\mathrm{Si}$ at low lattice temperatures. We consider electron-phonon scattering with intravalley and intervalley modes as a dominant mechanism for decay of nonequilibrium electrons in $\mathrm{Mg}^{\mathrm{O}}$ centers. The relaxation times are derived from pump-probe experiments measuring the decay of the transmission induced by changes in the populations of the probed $\mathrm{Mg}^{0}$ states subsequent to intracenter pumping and photoionization of $\mathrm{Mg}^{0}$ centers by radiation from an infrared free electron laser. The predicted relaxation rates range from $2 \times 10^{7}$ to about $7 \times 10^{10} \mathrm{~s}^{-1}$. The exact value depends on the binding energy between the lowest excited state and the $\mathrm{Mg}^{0}$ ground state. It should be noted that the slowest process may cause a bottleneck for intracenter relaxation. The longest intracenter relaxation step derived from our experimental data has a characteristic time of around $2 \mathrm{~ns}$, while the fastest step terminating in this long-lived state is about 270 ps. The longest observed lifetimes are significantly longer than for excited states of shallower donors in $\mathrm{Si}$ with the same parity. Capture of free electrons from the conduction band bottom has typical times larger than 850 ps.

\section{ELECTRON RELAXATION IN Si:Mg}

Photoexcited electrons decay towards their equilibrium state by radiative and nonradiative channels. The rates of spontaneous radiative decay are significantly lower than those 


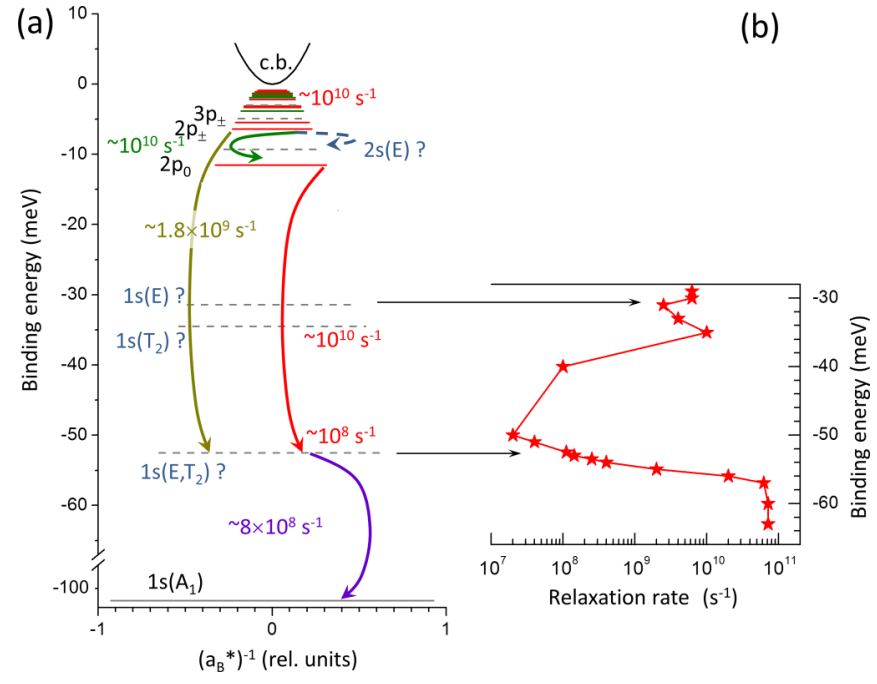

FIG. 1. (a) Scheme of the most probable paths for intracenter relaxation in $\mathrm{Si}: \mathrm{Mg}^{0}$ (energies according to [1]): discrete lines indicate positions of odd-parity (solid) and even-parity excited (dashed) states of $\mathrm{Mg}^{0}$ centers in the silicon band gap; $1 s\left(A_{1}\right)$ is the $\mathrm{Mg}^{0}$ ground state. The calculated relaxation rates for various positions of the $1 s\left(E, T_{2}\right)$ states are shown. (b) Calculated dependence of the $2 p_{0} \rightarrow 1 s\left(E, T_{2}\right)$ nonradiative relaxation rate on the binding energy of the $1 s\left(E, T_{2}\right)$ states.

for nonradiative processes as estimated using Fermi's golden rule. They are below $10^{5} \mathrm{~s}^{-1}$ for the $2 p_{ \pm} \rightarrow 1 s\left(A_{1}\right)$ transition and below $10^{4} \mathrm{~s}^{-1}$ for the $2 p_{0} \rightarrow 1 s\left(A_{1}\right)$ and $2 p_{0} \rightarrow 1 s(E)$ transitions. Therefore they can be neglected. Stimulated radiative decay requires population inversion between the analyzed states, which does not occur due to low optical pump rate.

Accurate theoretical calculations for nonradiative intracenter relaxation in $\mathrm{Si}_{\mathrm{Mg}} \mathrm{Mg}^{\mathrm{are}}$ difficult due to the unknown binding energies for all excited even-parity states of the $\mathrm{Mg}^{0}$ donor in $\mathrm{Si}$, which strongly contribute to intracenter decay. For the following calculations we assume the binding energy of the $1 s(E)$ ground split-off state to be $52.5 \mathrm{meV}$ as suggested by piezospectroscopy results [1]. The deepest excited state $1 s\left(T_{2}\right)$ should have a binding energy close enough to that of the $1 s(E)$ state and we neglect for simplicity the energy difference for these states, considering them as a single state $1 s\left(E, T_{2}\right)$ with the same binding energy.

We use the effective mass theory approach for the description of the $\mathrm{Mg}^{0}$ donor wave functions in $\mathrm{Si}$. The wave function of the ground state for an atom with two electrons (heliumlike) can be described by the Ritz variational method [11], in which the trial wave function is chosen as a product of the wave functions of each of the two bound electrons. The only interaction of the electrons which is considered is due to partial screening of the charge of the nucleus by these electrons. The eigenenergy has been taken from absorption spectroscopy data [1] and the eigenfunction has been calculated with the boundary condition of a quasiclassical asymptotic decay of the wave function far from the donor center as determined by the experimental value of the ground state energy [12]. The wave function of an excited state of a heliumlike atom [except the $1 s\left(E, T_{2}\right)$ state] was constructed by assuming that one bound, inner electron is in the ground state and causes screening of the charge of the nucleus, while the second outer electron populates an excited state and behaves as if it is in the potential field of a $\mathrm{Mg}$ atom with an effective positive charge +1 . Due to the large difference in the localization of the electron wave functions, the inner electron is not affected by the outer one.

To estimate the $\mathrm{Mg}^{0}$ states' lifetimes, scattering with both intravalley and intervalley phonons was taken into account. Intervalley scattering rates have been calculated using the deformation potential constants from [13]. The matrix element $M_{n m}^{l j}$ of the relaxation transition between the donor states labeled $n$ and $m$, which is accompanied by emission of an intervalley phonon, has been calculated according to [2]

$$
M_{n m}^{l j}=\sqrt{\frac{\hbar\left(D_{t} k\right)^{2}}{2 \rho V \omega_{q}}} \int F_{n}^{l^{*}}(r) F_{m}^{j}(r) e^{i\left(k-k^{\prime}-g-q\right) r} d r .
$$

Here $l$ and $j$ are the numbers of the equivalent conduction band valleys between whose the transition occurs, $\omega_{q}$ is the frequency of the emitted/absorbed phonon, $\rho$ is the silicon density, $V$ is the sample volume, $\boldsymbol{k}$ and $\boldsymbol{k}^{\prime}$ are the initial and final momenta, $\boldsymbol{q}$ is the phonon wave vector, $\boldsymbol{g}$ is the reciprocal lattice vector, $D_{t} k$ is the intervalley constant of the deformation potential [13], and $F_{m}^{j}(r)$ is the envelope of the wave function of the $m$ th state in the $j$ th valley. The matrix elements of the intervalley transitions between other types of states and matrix elements of intravalley transitions were treated in a similar way. The transition probability was calculated using Fermi's golden rule [2]:

$$
P_{n m}=\frac{2 \pi}{\hbar} K_{t} \sum_{q}\left|M_{n m}^{l j}\right|^{2} \delta\left(E_{n m}-\hbar \omega_{q}\right) .
$$

Here the coefficient $K_{t}$ ( $t=f, g$ is the type of interacting intervalley phonon) depends both on the symmetry of the distribution of wave functions among the valleys and on the type of the transition, $E_{n m}$ is the transition energy. The $2 p_{ \pm} \mathrm{Mg}^{0}$ state decays primarily into the $2 p_{0}$ state with the rate of about $10^{10} \mathrm{~s}^{-1}$ [Fig. 1(a)]. The relaxation rate of the $2 p_{0}$ state depends strongly on the energy gaps to the low lying valley-orbit-split states [Fig. 1(b)]. Assuming the binding energy for the $1 s\left(E, T_{2}\right)$ states to be about $52.5 \mathrm{meV}$, the decay of the $2 p_{0}$ and $2 p_{ \pm} \mathrm{Mg}^{0}$ states into the $1 s\left(E, T_{2}\right)$ states is governed by scattering with $f$-LA intervalley phonons. This yields values for the relaxation rates of approximately $10^{8}$ and $1.8 \times 10^{9} \mathrm{~s}^{-1}$, respectively [Fig. 1(a), solid arrows down]. If the $1 s\left(E, T_{2}\right)$ states are significantly shallower, the $2 p_{0}$ and $2 p_{ \pm} \mathrm{Mg}^{0}$ states could come into resonance with $f$-TA and $g$-LA intervalley phonons, similar to the case of shallow hydrogenlike donors in $\mathrm{Si}$ [3]. Therefore, in the latter case, both the $2 p_{0}$ and $2 p_{ \pm}$states would have total relaxation rates on the order of $10^{10} \mathrm{~s}^{-1}$ [Fig. 1(b)], i.e., as fast as their hydrogenlike analogs, having lifetimes of about 200 ps [5]. The $2 s \rightarrow 1 s$ processes, which are very efficient in intracenter relaxation for particular shallow donors [3,14], may not play such a significant role for double $\mathrm{Mg}$ donors due to the absence of one-phonon-impurity resonances. The common bottleneck in the relaxation process is the decay from the $1 s\left(E, T_{2}\right)$ states into the ground $\mathrm{Mg}^{0}$ state as it involves the largest energy gap. 
Such an energy gap can be overcome either by a two-phonon assisted process or by nonresonant phonons. An estimate of the latter type of interaction gives a rate of less than $10^{9} \mathrm{~s}^{-1}$.

\section{EXPERIMENTAL SETUP AND PROCEDURES}

\section{A. Sample preparation}

$\mathrm{Mg}$ impurities in Si may result in double-charge donor centers, which are known to occupy interstitial positions in the crystal lattice. However, in contrast to doping with shallower donor impurities — such as phosphorus or arsenicpreparation of bulk $\mathrm{Si}$ samples with a given concentration of $\mathrm{Mg}$ donors is a challenge. On the one side, this is related to the high volatility of $\mathrm{Mg}$ at conventional processing temperatures of Si wafers. On the other side, technologically important parameters, such as the solubility and the diffusion coefficient of $\mathrm{Mg}$ in $\mathrm{Si}$ at a given temperature, are not well known. For example, the highest concentration of $\mathrm{Mg}$ in Si has been reported as $2.6 \times 10^{15} \mathrm{~cm}^{-3}$ [15] achieved with a sandwich diffusion technique at $1350^{\circ} \mathrm{C}$ [1]. Values up to $1.5 \times 10^{19} \mathrm{~cm}^{-3}$ have been obtained for doping by liquid-phase epitaxy of Si from a SiMg melt at $1200^{\circ} \mathrm{C}$ [16].

The sandwich technique enables a relatively homogeneous distribution of interstitial $\mathrm{Mg}$ centers in bulk samples with a maximum concentration up to $2 \times 10^{15} \mathrm{~cm}^{-3}$ [1]. In order to meet the requirements of the present experiments, we have used a similar doping approach, namely diffusion of $\mathrm{Mg}$ in a Si wafer "sandwiched" between two other wafers with a focus on reaching a concentration of $\mathrm{Mg}^{0}$ centers at low temperature, which would be large enough to investigate intracenter relaxation by the pump-probe technique. It is particularly important to start with low-impurity Si crystals in order not to affect the optical properties of the samples with features which are due to other donor centers such as magnesium-oxygen $\mathrm{Mg}$-O complexes as well as singly ionized $\mathrm{Mg}^{+}$deep donors. $P$-type float-zone grown $\mathrm{Si}$ with a relatively low content of residual impurities and oxygen (the latter was less than $10^{16} \mathrm{~cm}^{-3}$ ) was used for the diffusion process. The resistivity of the initial material was in the range of 0.45 to $10 \mathrm{k} \Omega \mathrm{cm}$. Most experiments were performed on samples with an acceptor (predominantly boron) concentration less than $10^{13} \mathrm{~cm}^{-3}$. Mg films were deposited on all contacting surfaces of the sandwiched Si wafer by thermal evaporation of $\mathrm{Mg}$ in vacuum. Pure, $99.999 \% \mathrm{Mg}$ both in crystalline form and in pellets from different suppliers (Goodfellow, Mateck, GIRedMet, AMI) were used to dope the Si samples. The sandwiches were heated in sealed quartz ampoules in argon atmosphere or vacuum.

Different heating and cooling procedures have been tested in order to reach a high and spatially homogeneous interstitial Mg concentration. To check the homogeneity of the dopant distribution across the sample four-probe resistivity measurements were made at different points of the sample. Also, profiling of the resistivity along the depth of test samples was done. As a result, the appropriate regime for preparation of samples has been found to be heating at about $1200^{\circ} \mathrm{C}$ for about $1 \mathrm{~h}$ followed by rapid cooling of an ampoule with a flow of compressed air or nitrogen at room temperature. The welded auxiliary $\mathrm{Si}$ wafers covering a doped sample were then ground

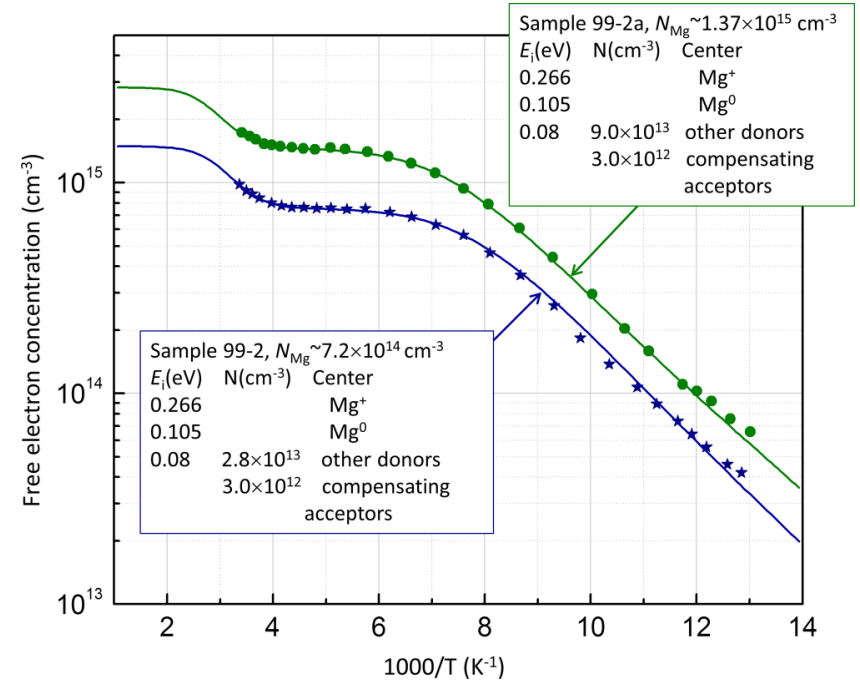

FIG. 2. Analysis of the impurity content in two Si samples after diffusion of Mg. The symbols are measured values from the Hall effect data. The insets show the sample parameters: concentrations of $\mathrm{Mg}$ in the interstitial state $\left(N_{\mathrm{Mg}}\right)$, ionization energies of neutral $\left(\mathrm{Mg}^{0}\right)$ and positively charged $\left(\mathrm{Mg}^{+}\right)$centers, concentration of unidentified donor centers, as well as the total compensating acceptor concentration. The sample parameters are determined from fitting the solution of the electroneutrality equation for the doped $n$-type $\mathrm{Si}$ (solid lines) to the experimental data (symbols).

off together with the original interface layer. When applying the described procedure, samples with a final thickness of about $1 \mathrm{~mm}$ were fabricated.

The results of the doping procedure were evaluated by infrared absorption spectroscopy (at $T \sim 5 \mathrm{~K}$ ) and temperaturedependent Hall effect measurements (in the range of 78 $300 \mathrm{~K})$. From the latter it was possible to determine the concentration of the introduced deep donor $\mathrm{Mg}$ centers and their compensation by acceptors. The procedure is similar to that applied for the sulfur deep donor in Si [17] and includes fitting of the solution of the electroneutrality equation [18] to the set of Hall data points. Peculiarities of statistics of the $n$-type semiconductor doped with double donor deep impurity $[19,20]$ were taken into account, when solving the electroneutrality equation. Typical data for the free electron concentration in samples $n$ obtained from Hall effect measurements along with results of fitting calculated $n(T)$ curves to the sets of experimental points are shown in Fig. 2. The agreement of the experimental and calculated $n(T)$ dependencies was obtained through variation of the density of interstitial $\mathrm{Mg}$ centers, while the concentration of acceptors was taken as the content of the initial $p$-type silicon. Also, a small density of relatively shallow donor centers, see Fig. 2, was taken into account in the electroneutrality equation. Typical data of the free electron concentration $n$ obtained from temperature-dependent Hall measurements are shown in Fig. 2 along with a numerical solution of the electroneutrality equation for $n$-type Si doped with $\mathrm{Mg}$. One can see that the temperature dependencies of the equilibrium concentration of free electrons in the measured samples are satisfactorily described by the statistics of double-charge donor centers that are partly compensated by acceptors. We point out that the ground state binding energies 


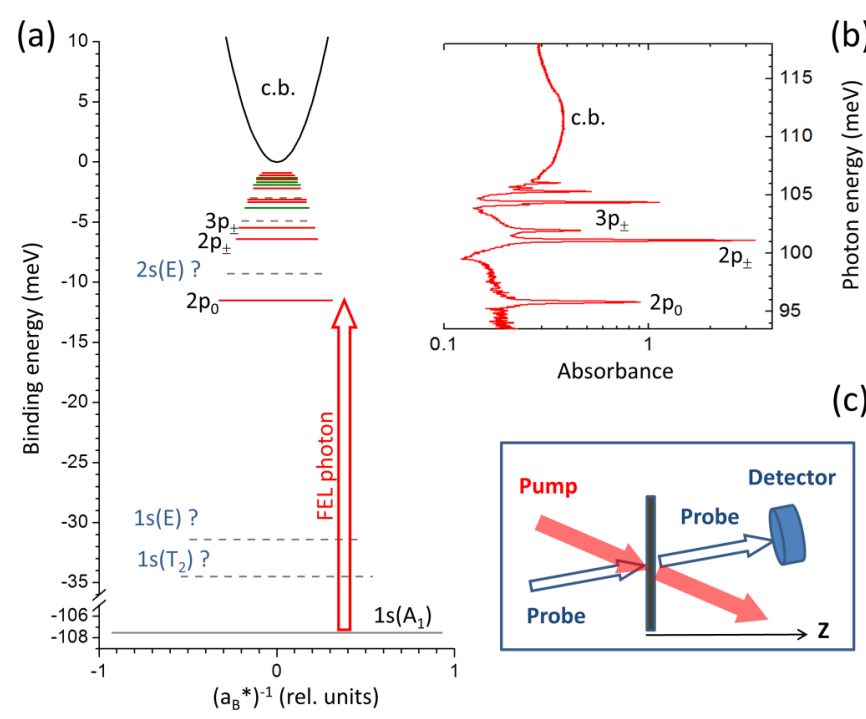

FIG. 3. (a) Optical excitation of bound electrons in the pumpprobe experiment on the band diagram of magnesium impurity levels. (b) Absorption spectrum of a Si:Mg sample in the range of $\mathrm{Mg}^{0}$ transitions. The marked states show the transition terminators. (c) Principle of the pump-probe experiment; pump and probe pulses have an incident angle on a sample of about $10^{\circ}$.

of subsequent ionization of deep donors (in our case $\mathrm{Mg}^{0}$ and $\mathrm{Mg}^{+}$) that are derived from fitting the theoretical dependence to the experimental data are in good agreement with data obtained by infrared absorption spectroscopy [Fig. 3(b)].

This fact permits us to conclude that the concentration of interstitial $\mathrm{Mg}$ is correctly determined by this method. In addition, the density of compensating acceptors in the doped samples is close to the boron concentration in the initial $p$-type Si. It was found, however, that the doping procedure introduces some more shallow donors that cannot be identified from the Hall data obtained at rather high temperature. In the optical experiments we used those samples with the highest $\mathrm{Mg}^{0}$ concentration, which-according to estimates from the absorption spectra and Hall data-was in the range $(1.1-1.3) \times 10^{15} \mathrm{~cm}^{-3}$. Samples with dimensions of $7 \times 7 \times 1 \mathrm{~mm}^{3}$ wedged with $0.2^{\circ}-0.5^{\circ}$ were prepared from the doped wafers. The large facets were polished. No degradation of the infrared absorption by $\mathrm{Mg}^{0}$ was observed after storing the samples for 1.5 years at room temperature. This observation is in contradiction with the results reported in [1], where a strong decay of the absorption was found when repeating the measurements after 19 months. The difference in the behavior between the samples might be related to the different methods of cooling after diffusion, where the samples of [1] were quenched with liquid nitrogen. Such strong quenching could freeze a large density of intrinsic defects in the Si lattice, which could favor subsequent solid-state reactions even at room temperature.

In all of the $\mathrm{Mg}$-doped $\mathrm{Si}$ samples a certain concentration of lithium interstitial centers, which reached $10^{14} \mathrm{~cm}^{-3}$ in some samples, was detected by infrared absorption spectroscopy. We suppose that the lithium centers are responsible for the shallow donor levels that could not be precisely identified by the Hall measurements (Fig. 2). Replication of the complete doping procedure at the same conditions but with reference Si samples without diffusion of $\mathrm{Mg}$ does not show lithium related lines in the infrared absorption spectra. Therefore, we assume that the presence of lithium interstitial donors in the Mg-doped samples is due to residual lithium in the purchased magnesium. Although residual elements were specified to be below $0.0001 \%$, the high solubility and high saturation concentration of lithium in Si permit the occurrence of lithium donors at detectable levels.

\section{B. Pump-probe experiment}

Recombination times of particular electronic states can be directly measured in the time domain using a pump-probe technique [Fig. 3(c)]. This requires the irradiation of the sample with a short optical pulse. The excitation photon energy corresponds to the energy of the probed electronic transition [Fig. 3(a)]. The pulse duration must be much shorter than the characteristic times of the recombination processes involved and a time window between pump and probe pulses has to cover the range (at least better than $1 / e$ decay) of characteristic times. Once this is satisfied, the probe pulse measures the change of the complex refractive index (usually absorption), induced by a pump pulse after an adjustable delay.

The measurements were carried out with dedicated pumpprobe experimental setups at two different infrared free electron laser (FEL) facilities: FELIX, Radboud University, Nijmegen, The Netherlands, and FELBE, Helmholtz-Zentrum Dresden-Rossendorf, Germany. Details of the experimental setups there are given elsewhere [6,21]. In both cases the $\mathrm{Si}$ samples were cooled in a liquid helium flow cryostat down to $5 \mathrm{~K}$. The temporal resolution of both systems ranges from about 10 ps (a typical FEL micropulse duration) to about $1.5 \mathrm{~ns}$ (optical path in the delay lines). The pump-probe setups rely on different detection approaches, namely a zeroing compensation scheme for each probe micropulse with a fast photoconducting detector (FELIX) and a lock-in detection scheme with a mechanically chopped FEL beam for the quasicontinuous FEL mode at FELBE. Both pump and probe beams had spots of about $2 \mathrm{~mm}$ diameter on the sample in the FELIX setup, while the probe beam was focused to about $400 \mu \mathrm{m}$ in the FELBE setup. Spectral width of the FEL signal measured as half-width at half-maximum was better than $1 \%$ of the beam wavelength.

\section{Data analysis}

Assuming an instantaneous excitation $I(t=0)$ by the pump pulse, which is approximately constant in the probed sample, i.e., $\alpha(t) d \ll 1[\alpha(t)$ is the absorption coefficient and $d$ is the thickness of the sample], and neglecting multiple reflections in the sample, the normalized pump-probe signal $S(t)$ can be written as the change of the probe transmission $T(t)$ [21]:

$$
S(t)=\frac{T(t)-T_{0}(t)}{T_{0}(t)} \approx e^{-\Delta \alpha(t) d}-1=e^{-\sigma \Delta N(t) d}-1,
$$

where $T_{0}(t)$ is the probe transmission without pump pulse, $\Delta \alpha(t)=\sigma \Delta N(t)$ is the pump-induced change of the absorption coefficient of the probed optical transistion, $\Delta N(t)$ is the pump-induced change of the state population difference, and $\sigma$ is the absorption cross section of a center at the 
pump-probe photon energy. In the case of weak absorption, the measured pump-probe signal is directly proportional to the population difference $S(t) \approx \sigma \Delta N(t) d$ and the characteristic times of $S(t)$ reflect the lifetime of the probed excited state and the recovery of the ground state population. The decay times derived from the pump-probe experiment remain almost independent on pump powers up to about $20 \mathrm{~nJ}$ per micropulse. Since a real electronic system is inherently a multilevel one, additional levels can contribute to the pump-probe signal. These contributions can be extracted by solving the balance equations of a multilevel system.

\section{RELAXATION OF THE EXCITED STATES IN Si:Mg}

\section{A. Relaxation of the $2 p_{0}$ state in $\mathrm{Si}: \mathrm{Mg}^{0}$}

To study the intracenter relaxation, $\mathrm{Si}: \mathrm{Mg}$ samples were excited with light resonant to the dipole allowed transitions originating from the $\mathrm{Mg}^{0}$ ground state $1 s\left(A_{1}\right)$. The observed temporal dependences of the probe transmission indicate the existence of a component with a rather large characteristic decay time, which is at the limit of the available time coverage of the pump-probe setup [Fig. 4(a)].

Relaxation of the lowest odd-parity $2 p_{0}$ state is the simplest relaxation process in $\mathrm{Si}: \mathrm{Mg}^{0}$. A reasonable approach to describe this process is based on a three-level system [staircase relaxation $\left.2 p_{0} \rightarrow 1 s\left(E, T_{2}\right) \rightarrow 1 s\left(A_{1}\right)\right]$ with dominant relaxation steps between adjacent levels [i.e., $2 p_{0} \rightarrow 1 s\left(E, T_{2}\right)$ and $\left.1 s\left(E, T_{2}\right) \rightarrow 1 s\left(A_{1}\right)\right]$. Direct nonradiative relaxation in the ground state is negligible and not considered in the data analysis. We solve a system of corresponding balance equations for the populations $n_{1}, n_{2}, n_{3}$ of the involved levels; assuming $n_{1}+n_{2}+n_{3}=N$, where $N$ is the total concentration of bound electrons and the indices refer to the respective cascade under discussion, here 1, 2, and 3 refer to the $1 s\left(A_{1}\right), 1 s\left(E, T_{2}\right)$, and $2 p_{0}$ states, respectively. Assuming (a)

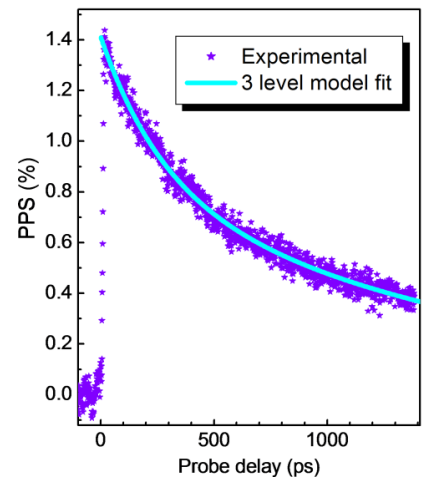

(b)

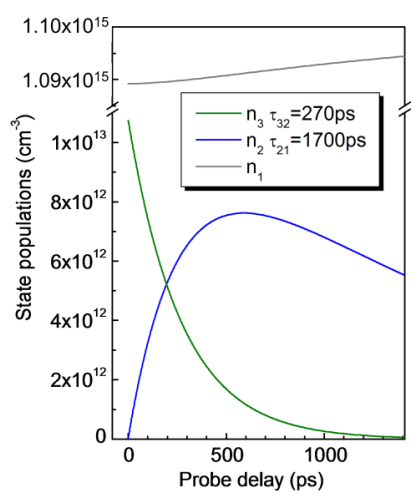

FIG. 4. Pumping the $\mathrm{Mg}^{0} 1 s\left(A_{1}\right) \rightarrow 2 p_{0}$ transition. (a) Typical behavior of pump-probe signal and a three-level model [staircase relaxation $\left.2 p_{0} \rightarrow 1 s\left(E, T_{2}\right) \rightarrow 1 s\left(A_{1}\right)\right]$ fit. The FEL pump wavelength was about $13 \mu \mathrm{m}(\sim 95 \mathrm{meV})$. The pump micropulse energy is $20 \mathrm{~nJ}$. The sample parameters were: $N\left(\mathrm{Mg}^{0}\right) \approx 1.1 \times 10^{15} \mathrm{~cm}^{-3}$, $N(\mathrm{Li}) \approx 1.1 \times 10^{14} \mathrm{~cm}^{-3}$. (b) Population dynamics as derived in the frame of the three-level model with parameters defined from the best fit to the experimental data. $n_{2}(0)=0$, the solutions for $t \geqslant 0$ are

$$
\begin{aligned}
& n_{3}(t)=n_{3}(0) e^{-W_{32} t}, \\
& n_{2}(t)=n_{3}(0) \frac{W_{32}}{W_{21}-W_{32}}\left(e^{-W_{32} t}-e^{-W_{21} t}\right), \\
& n_{1}(t)=N-n_{3}(0) \frac{1}{W_{21}-W_{32}}\left(W_{21} e^{-W_{32} t}-W_{32} e^{-W_{21} t}\right),
\end{aligned}
$$

$n_{1}(t), n_{2}(t)$, and $n_{3}(t)$ refer to the time-dependent populations of the $1 s\left(A_{1}\right)$ ground state, the $1 s\left(E, T_{2}\right)$ states, and the $2 p_{0}$ state, respectively. $n_{3}(0)$ is the initial (at $t=0$ ) population of the probed level, i.e., $2 p_{0}$ state. It depends on the pump photon flux density and the cross section $\sigma_{13}$ of the transition $1 \rightarrow 3$. $W_{32}$ and $W_{21}$ are the relaxation rates for the $3 \rightarrow 2\left[2 p_{0} \rightarrow\right.$ $\left.1 s\left(E, T_{2}\right)\right]$ and $2 \rightarrow 1\left[1 s\left(E, T_{2}\right) \rightarrow 1 s\left(A_{1}\right)\right]$ transitions. The pump-probe signal is then

$$
S(t)=\frac{T(t)-T_{0}(t)}{T_{0}(t)} \approx e^{-\sigma_{13}\left(n_{1}(t)-n_{3}(t)-N\right) d}-1 .
$$

Note here that the time-dependent part of the pump-probe signal in Eq. (5) depends on the characteristic relaxation rates $W_{32}$ and $W_{21}$ and these rates have a fixed ratio. It should be noted that the three-level model includes an analysis of the population of the involved levels as well as pump intensities through $n_{3}(0)$, which is proportional to the pump intensity. We fit the experimental data with Eq. (5). There are three fit parameters: $W_{32}, W_{21}$, and the initial population $n_{3}(0)$. Typical results are shown in Fig. 4. This fit yields $\tau_{32}=W_{32}^{-1} \approx$ $270 \pm 10 \mathrm{ps}$ and $\tau_{21}=W_{21}^{-1} \approx 1700 \pm 50 \mathrm{ps}$ and a pump rate, which provides the relative population of the excited $2 p_{0}$ level $n_{3} / N$ as $1 \%$ at time "zero" (zero probe delay corresponds to overlapping pump and probe pulses). At these parameters, the relative population of the "metastable" excited $1 s\left(E, T_{2}\right)$ level $n_{2} / N$ reaches its maximum value of $0.7 \%$ after about $600 \mathrm{ps}$ and then decays before the next pump pulse. This low population confirms the assumption of low excitation regime and the absence of an inverted electron distribution for the transition of interest. Thus, the analysis indicates the existence of a long-living, significantly longer than $1 \mathrm{~ns}$, excited state in Si: $\mathrm{Mg}^{0}$. The three-level model shows that such a state must be an intermediate one in the $2 p_{0} \rightarrow 1 s\left(E, T_{2}\right) \rightarrow 1 s\left(A_{1}\right)$ cascade, i.e., the valley-orbit-split $1 s(E)$ or $1 s\left(T_{2}\right)$ state. The first relaxation step $2 p_{0} \rightarrow 1 s\left(E, T_{2}\right)$ goes with a characteristic time of about $270 \mathrm{ps}$, while the second step $1 s\left(E, T_{2}\right) \rightarrow$ $1 s\left(A_{1}\right)$ is much longer (approximately $1700 \mathrm{ps}$ ). It should be noted that even the first relaxation step is about 50\% longer than those for shallow donors in silicon [6].

\section{B. Relaxation of the $2 p_{ \pm}$state in $\mathrm{Si}: \mathrm{Mg}^{\mathbf{0}}$}

For these experiments the FEL was tuned to $12.4 \mu \mathrm{m}$ (pumping the $2 p_{ \pm}$state). Relaxation of the $2 p_{ \pm}$state [Fig. 5(a)] is a more complex process which may have different relaxation paths with a first decay step in the $2 p_{0}$ state (theoretical estimate is $\left.10^{10} \mathrm{~s}^{-1}\right)$, in the $1 s\left(E, T_{2}\right)$ state (theoretical estimate is $1.8 \times 10^{9} \mathrm{~s}^{-1}$ assuming the latter binding energy to be $52.5 \mathrm{meV}$ ), and in the $2 s$ state (unknown state energy and corresponding decay rate). The relaxation stair via the $2 s$ state $2 p_{ \pm} \rightarrow 2 s \rightarrow 1 s$ is the fastest for the particular shallow hydrogen donors in silicon [14], but cannot be accurately 
(a)

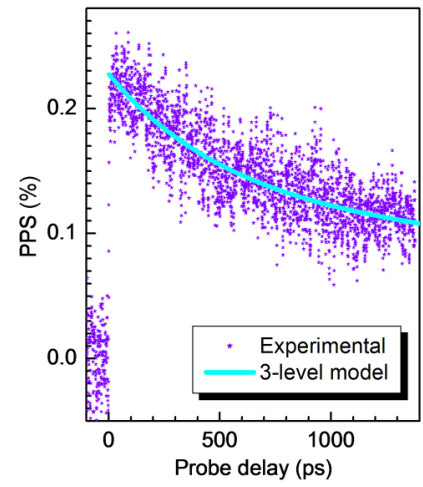

(b)

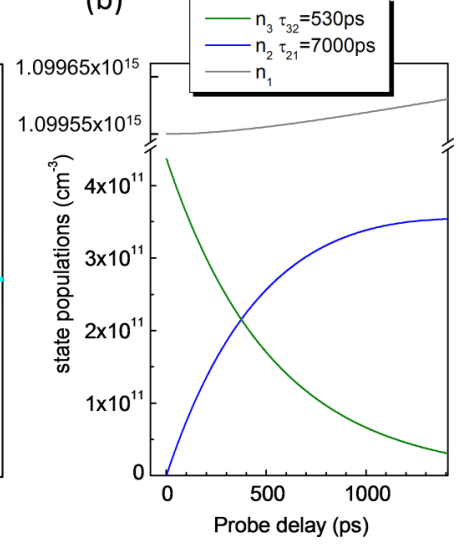

FIG. 5. Pumping the $\mathrm{Mg}^{0} 1 s\left(A_{1}\right) \rightarrow 2 p_{ \pm}$transition. (a) Typical behavior of pump-probe signal and a three-level model [staircase relaxation $\left.2 p_{ \pm} \rightarrow 1 s\left(E, T_{2}\right) \rightarrow 1 s\left(A_{1}\right)\right]$ fit. The FEL pump wavelength is about $12.4 \mu \mathrm{m}(\sim 100 \mathrm{meV})$. The pump micropulse energy was $6.7 \mathrm{~nJ}$. (b) Population dynamics as derived in the frame of the three-level model. The Si:Mg sample was from the same material as in Fig. 4.

estimated for Si: $\mathrm{Mg}^{0}$ because of absent knowledge of the binding energy for all involved even-parity $\mathrm{Mg}^{0}$ excited states. This channel can contribute significantly to the relaxation of the $2 p_{ \pm}$state for the case of the relatively deep $1 s\left(E, T_{2}\right)$ state. For the channel via the $1 s\left(E, T_{2}\right)$ state a three-level model can give again a reasonable description as a two-step process $2 p_{ \pm} \rightarrow 1 s\left(E, T_{2}\right) \rightarrow 1 s\left(A_{1}\right)$. A three-level model fit for the $2 p_{ \pm}$state [Fig. $\left.5(\mathrm{a})\right]$ gives times of $\tau_{32}=W_{32}^{-1} \approx$ $530 \pm 30 \mathrm{ps}$ and $\tau_{21}=W_{21}^{-1} \approx 7000 \pm 300 \mathrm{ps}$ and a maximum population of the $2 p_{ \pm}$state of $n_{3}(0) / N \approx 4 \times 10^{-4}$. Note that the value $W_{32} \approx 1.88 \times 10^{9} \mathrm{~s}^{-1}$ resulting from this fit corresponds well to the theoretical estimation assuming the larger value of the binding energy of the state 2 [i.e., $1 s\left(E, T_{2}\right)$ ]. Assuming the first relaxation step to occur into the $2 p_{0}$ state, an obvious description of the three-step process $2 p_{ \pm} \rightarrow$ $2 p_{0} \rightarrow 1 s\left(E, T_{2}\right) \rightarrow 1 s\left(A_{1}\right)$ is a four-level system with the known last two-steps cascade. For the latter case we solve a system of corresponding balance equations for populations $n_{1}, n_{2}, n_{3}, n_{4}$ of the involved levels $n_{1}+n_{2}+n_{3}+n_{4}=N$. The corresponding solutions for the populations assuming $n_{3}(0)=0, n_{2}(0)=0$ are

$$
\begin{aligned}
n_{4}(t)= & n_{4}(0) e^{-W_{43} t}, \\
n_{3}(t)= & n_{4}(0) \frac{W_{43}}{W_{32}-W_{43}}\left(e^{-W_{43} t}-e^{-W_{32} t}\right), \\
n_{2}(t)= & n_{4}(0) \frac{W_{32} W_{43}}{W_{32}-W_{43}}\left(\frac{e^{-W_{43} t}}{W_{21}-W_{43}}\right. \\
& \left.-\frac{e^{-W_{32} t}}{W_{21}-W_{32}}-\frac{e^{-W_{21} t}}{W_{32}}\right), \\
n_{1}(t)= & N-n_{2}(t)-n_{3}(t)-n_{4}(t),
\end{aligned}
$$

where $n_{4}(0)$ is the initial (at $t=0$ ) population of the $2 p_{ \pm}$ state, $W_{43}$ is the relaxation rate for the $4 \rightarrow 3$ transition. The

(a)

(b)
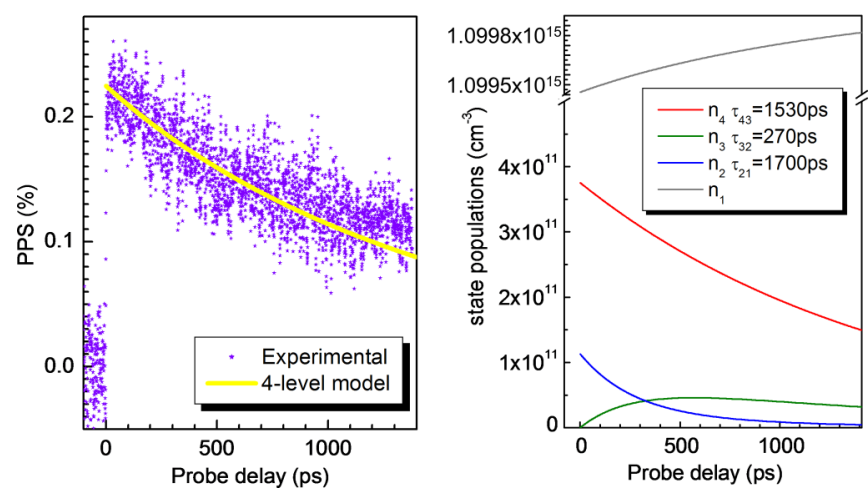

FIG. 6. Pumping the $\mathrm{Mg}^{0} 1 s\left(A_{1}\right) \rightarrow 2 p_{ \pm}$transition. (a) Typical behavior of the pump-probe signal and a four-level model [staircase relaxation $\left.2 p_{ \pm} \rightarrow 2 p_{0} \rightarrow 1 s\left(E, T_{2}\right) \rightarrow 1 s\left(A_{1}\right)\right]$ fit with two relaxation times fixed as determined in the fit for the $2 p_{0}$ state in Fig. 4(b). (b) Population dynamics as derived in the frame of the four-level model.

pump-probe signal is then

$$
S(t)=\frac{T(t)-T_{0}(t)}{T_{0}(t)} \approx e^{-\sigma_{14}\left[n_{1}(t)-n_{4}(t)-N\right] d}-1 .
$$

Fixing the relaxation from the $2 p_{0}$ state as it was determined before to $\tau_{32}=W_{32}^{-1} \approx 270 \mathrm{ps}$ and $\tau_{21}=W_{21}^{-1} \approx 1700 \mathrm{ps}$, we get for the pumping case in Fig. 5(a) the best fit for the lifetime of the $2 p_{ \pm}$state $\tau_{43}=W_{43}^{-1} \approx 1530 \pm 130 \mathrm{ps}$ and a maximum population of the $2 p_{ \pm}$state of $n_{4}(0) / N \approx 4 \times 10^{-4}$ [Fig. 6(b)]. It should be noted that the fit with a four-level model with the fixed cascade $2 p_{0} \rightarrow 1 s\left(E, T_{2}\right) \rightarrow 1 s\left(A_{1}\right)$ is worse than one with a three-level model cascade $2 p_{ \pm} \rightarrow 1 s\left(E, T_{2}\right) \rightarrow$ $1 s\left(A_{1}\right)$. One can see clearly that the chosen four-level model for the decay of the $2 p_{ \pm}$state results in the large deviation of the fit to the experimental data at long delays is obvious and requires significantly larger time $\tau_{21}$ or significantly shorter time $\tau_{43}$. Indeed, the shorter than $1530 \mathrm{ps}$ time is required to fit properly the experimental data in the front of the pump-probe signal in both models, which it turn, interferes with a proper fit at longer times if we fix the three-level cascade $2 p_{0} \rightarrow$ $1 s\left(E, T_{2}\right) \rightarrow 1 s\left(A_{1}\right)$ in the four-level model. Thus, we ascribe a characteristic time of about 530 ps required for accurate fitting of the experimental data in the three-level model to the $2 p_{ \pm}$state of $\mathrm{Mg}^{0}$ center in Si. For both models the accuracy for the determination of a characteristic time for the longtime component suffers from insufficient data for the probe delays longer than $1.5 \mathrm{~ns}$, the physical limit of the stage in our experiment. Note here that this time is longer than the theoretically predicted one for the $2 p_{ \pm} \rightarrow 2 p_{0}$ relaxation time $\left(10^{10} \mathrm{~s}^{-1}\right)^{-1}=100 \mathrm{ps}$ (Fig. 1).

\section{FREE ELECTRON CAPTURE IN Si:Mgo}

Capture of free electrons, which are excited into the conduction band, is also a complex process [Fig. 7(a)]. At the same time characteristic times for both relaxations are very similar and clearly longer than those for the relaxation process 
(a)

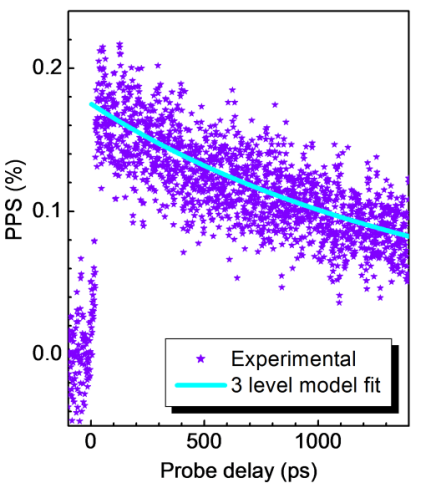

(b)

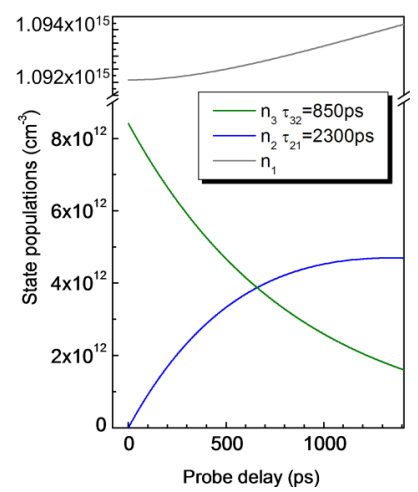

FIG. 7. Pumping the $\mathrm{Mg}^{0}$ continuum. (a) Typical pump-probe signal and a three-level model fit. The FEL wavelength is $11 \mu \mathrm{m}$ $(\sim 113 \mathrm{meV}$ ) and the pump micropulse energy is $1 \mathrm{~nJ}$. The Si:Mg sample was from the same material as in Fig. 4. (b) Population dynamics as derived in the frame of the three-level model.

from the $2 p_{0}$ state. For these experiments the FEL was tuned to $11 \mu \mathrm{m}$ (pumping into the conduction band).

We simplify a model for analysis assuming that the intermediate excited states can be effectively described by a metastable level. This effective three-level model gives $\tau_{32}=$ $W_{32}^{-1} \approx 850 \pm 10 \mathrm{ps}$ and $\tau_{21}=W_{21}^{-1} \approx 2300 \pm 50$ ps with the relative population $n_{3} / N$ below $1 \%$ [Fig. 7(b)].

\section{SUMMARY}

In summary, we have studied the dynamics of electrons bound to neutral $\mathrm{Mg}$ centers in $\mathrm{Si}$ at low lattice temperatures using the pump-probe technique. The measurements could be carried out at very low pump rates, which provide the relative populations of the excited $2 p_{0}$ and $2 p_{ \pm}$states below $10^{-3}$ and below $1 \%$ for the case of photoionization in the continuum. The data were analyzed in the frame of a three-level and four-level relaxation model. The main result from these experiments is that the shorter time $\left(\sim 270 \mathrm{ps}\right.$ for the $2 p_{0}$ state and $\sim 530$ ps for the $2 p_{ \pm}$state) characterizes the first step of the relaxation of excited electrons while the longer times (about $1.7 \mathrm{~ns}$ ) indicate the existence of a metastable state, very

likely the lowest excited state of the $\mathrm{Mg}^{0}$ center. The capture of photoionized electrons takes at least $2 \mathrm{~ns}$ for recovery of optical transmission in $\mathrm{Si}: \mathrm{Mg}^{0}$. The common bottleneck is apparently determined by the last relaxation step between the lowest excited valley-orbit-split $1 s(E)$ or/and $1 s\left(T_{2}\right)$ state and the $\mathrm{Mg}^{0}$ ground state. The reason for such a long lifetime constant is the large energy gap of this step and absent resonances with principal phonons in $\mathrm{Si}$, enhancing such a relaxation process. Other dynamical characteristics for $\mathrm{Mg}$ donors corresponding to transition from odd-parity state(s) into the $1 s(E)$ and/or the $1 s\left(T_{2}\right)$ states are somewhat (by about $50 \%$ ) slower but of the same order of magnitude as those found for more shallow hydrogenlike donor centers in $\mathrm{Si}$, where impurity-singlephonon resonances dominate intracenter relaxation. Accurate theoretical calculations are constrained due to missing data on the binding energies of even-parity excited states, which may play an active role in intracenter relaxation. The remaining discrepancy to the theoretical estimates, in particular for the long relaxation time, is due to two main factors: complex decay routes, which are not properly described by the simplified models, and the limited set of data at large probe delay, making the fit procedure less accurate. Relaxation times derived from the experimental data together with the theoretical estimates for the relaxation rates do not constrain the binding energy of the valley-orbit-split $1 s\left(E, T_{2}\right)$ states. These missing data require additional nonequilibrium and/or Raman spectroscopy of Si:Mg.

\section{ACKNOWLEDGMENTS}

This work has been partly supported by the joint GermanRussian program "Research on technological advances of radiation sources of photons and neutrons based on accelerators and neutron sources in cooperation with research organizations and universities of the Federal Republic of Germany" (InTerFEL project, BMBF No. 05K2014 and the Russian Ministry of Science and Education No. RFMEFl61614X0008) as well as the Russian Foundation for Basic Research (project 14-02-00638). N.D. and A.P. gratefully acknowledge support by the Helmholtz Research School on Security Technologies. We are grateful to the FELBE and FELIX teams for their dedicated support.
[1] L. T. Ho and A. K. Ramdas, Phys. Rev. B 5, 462 (1972).

[2] V. V. Tsyplenkov, E. V. Demidov, K. A. Kovalevsky, and V. N. Shastin, Semiconductors 42, 1016 (2008).

[3] V. Tyuterev, J. Sjakste, and N. Vast, Phys. Rev. B 81, 245212 (2010).

[4] M. Kleverman, H. G. Grimmeiss, A. Litwin, and E. Janzen, Phys. Rev. B 31, 3659 (1985).

[5] N. Q. Vinh, P. T. Greenland, K. Litvinenko, B. Redlich, A. F. G. van der Meer, S. A. Lynch, M. Warner, A. M. Stoneham, G. Aeppli, D. J. Paul, C. R. Pidgeon, and B. N. Murdin, PNAS 105, 10649 (2008).

[6] H.-W. Hübers, S. G. Pavlov, S. A. Lynch, Th. Greenland, K. L. Litvinenko, B. Murdin, B. Redlich, A. F. G. van der Meer, H. Riemann, N. V. Abrosimov, P. Becker, H.-J. Pohl,
R. Kh. Zhukavin, and V. N. Shastin, Phys. Rev. B 88, 035201 (2013).

[7] B. Pajot, Optical Absorption of Impurities and Defects in Semiconducting Crystals: I. Hydrogen-like Centres (Springer, Berlin, 2010).

[8] F. A. Johnson, Proc. Phys. Soc. 73, 265 (1959).

[9] P. A. Temple and C. E. Hathaway, Phys. Rev. B 7, 3685 (1973).

[10] V. N. Shastin, V. V. Tsyplenkov, R. Kh. Zhukavin, K. A. Kovaleskii, Yu. A. Astrov, H.-W. Hübers, and S. G. Pavlov, in Proceedings of the XVIII Symposium on Nanophysics \& Nanoelectronics (IPM, Nizhny Novgorod, 2014), p. 678.

[11] H. A. Bethe and E. E. Salpeter, Quantum Mechanics of Oneand Two-Electron Atoms (Springer, Berlin, 1957). 
[12] B. I. Shklovskii and A. L. Efros, Electronic Properties of Doped Semiconductors, Springer Series in Solid-State Sciences, Vol. 45 (Springer, Berlin, 1984).

[13] C. Jacoboni and L. Reggiani. Rev. Mod. Phys. 55, 645 (1983).

[14] S. G. Pavlov, H.-W. Hübers, P. M. Haas, J. N. Hovenier, T. O. Klaassen, R. Kh. Zhukavin, V. N. Shastin, D. A. Carder, and B. Redlich, Phys. Rev. B 78, 165201 (2008).

[15] A. Lin, J. Appl. Phys. 53, 6989 (1982).

[16] H. Sigmund, J. Electrochem. Soc. 129, 2809 (1982).
[17] Yu. A. Astrov, L. M. Portsel, A. N. Lodygin, and V. B. Shuman, Semicond. Sci. Technol. 26, 055021 (2011).

[18] J. S. Blakemore, Semiconductor Statistics (Pergamon, Oxford, 1962).

[19] H. Brooks, Adv. Electron. Electron Phys. 7, 85 (1955).

[20] D. C. Look, Phys. Rev. B 24, 5852 (1981).

[21] N. Deßmann, S. G. Pavlov, V. N. Shastin, R. Kh. Zhukavin, V. V. Tsyplenkov, S. Winnerl, M. Mittendorff, N. V. Abrosimov, H. Riemann, and H.-W. Hübers, Phys. Rev. B 89, 035205 (2014). 